\title{
Lack of Association between Brain-Derived Neurotrophic Factor Gene Val66Met Polymorphisms and Generalized Social Anxiety Disorder in Korean Population
}

\author{
Jin-Sung Park', Sewon Lim', Juwon $\mathrm{Ha}^{1}$, Min-Soo Lee ${ }^{2}$, Kang-Seob Oh${ }^{1}$ \\ Department of Psychiatry, 'Kangbuk Samsung Hospital, Sungkyunkwan University School of Medicine, ${ }^{2}$ Korea University College of \\ Medicine, Seoul, Korea
}

\begin{abstract}
Objective: Several lines of evidence suggest that brain-derived neurotrophic factor (BDNF) plays a role in the pathophysiology of anxiety. We analyzed the association of the BDNF gene polymorphism, G196A (val66met), in the coding region of exon XIIIA in chromosome 11p13, and generalized social anxiety disorder (GSAD).

Methods: Patients with GSAD ( $n=73$ ) and age-matched control subjects ( $n=152$ ) were tested for the BDNF (val66met) polymorphism. A clinical interview and a Mini-International Neuropsychiatric Interview were conducted by trained psychiatrists in order to diagnose GSAD. The symptomatic characteristics of the GSAD patients were assessed with the Hamilton Anxiety Rating Scale, the Beck Anxiety Inventory, the Retrospective Self Report of Inhibition, the Spielberger State-Trait Anxiety Inventory, and the Liebowitz Social Anxiety Scale.

Results: There were no significant differences in the frequencies of the genotypes ( $\chi^{2}=0.961$, degree of freedom $\left.[\mathrm{df}]=2, p=0.619\right)$, alleles ( $\chi^{2}=0.415, \mathrm{~d} f=1, p=0.519$ ), or allele (methionine) carriers $\left(\chi^{2}=0.019, \mathrm{~d} f=1, p=0.889\right.$ ) between the patients and controls. In addition, when we compared the severity of social anxiety symptom as determined by the clinical scales with the genotypes of the BDNF gene, we could not find any significant differences between the genotypes or allele carriers.

Conclusion: These results do not support the hypothesis that the BDNF gene might be a candidate gene for susceptibility or severity of GSAD in the Korean population in this study.
\end{abstract}

KEY WORDS: Social anxiety disorder; Brain-derived neurotrophic factor; Polymorphism.

\section{INTRODUCTION}

The prevalence of social anxiety disorder (SAD), which is a common anxiety disorder that is characterized by excessive fear and anxiety in response to one or more social or performance situations, has been reported to be $12-14 \%$ of the general population. ${ }^{1,2)} \mathrm{SAD}$ has a chronic course, and it therefore can be a significant and sometimes disabling burden to patients. It is associated with functional impairment, ${ }^{3)}$ a high economic cost, ${ }^{4)}$ and low quality of life. ${ }^{5)}$ According to the Diagnostic and Statistical Manual of Mental Disorders, revision IV (DSM-IV), generalized social anxiety disorder (GSAD), is a subtype of SAD which refers to people who fear most social situ-

Received: August 4, 2011 / Revised: November 3, 2011

Accepted: November 14, 2011

Address for correspondence: Kang-Seob Oh, MD, PhD

Department of Psychiatry, Kangbuk Samsung Hospital,

Sungkyunkwan University, 108 Pyung-dong, Chongro-gu, Seoul

110-746, Korea

Tel: +82-2-2001-2478, Fax: +82-2-2001-2211

E-mail: ks2485@empal.com ations. ${ }^{6}$ Compared to the non-generalized SAD subtype, GSAD has an earlier age of onset and is associated with greater functional impairment. ${ }^{7)}$

Family studies of subjects with SAD have reported that a high percentage of SAD is observed in first-degree relatives of patients. ${ }^{8)}$ Especially in first-degree relatives of GSAD patients, the prevalence of GSAD was 10 times higher compared to the general population and familial social phobia is known to be more common among patients with GSAD than patients with non-generalized SAD, so that it is considered a familial form of the disorder. ${ }^{9}$ Although there are no previous genetic studies comparing GASD to non-generalized SAD, these findings and clinical characteristics of GSAD suggest that GSAD may have a significant genetic predisposition.

Brain-derived neurotrophic factor (BDNF) is the most abundant neurotrophin in the brain. It has been recognized that BDNF plays an important role in neuronal growth and differentiation during development, and it contributes to the survival, function, and plasticity of neurons in adulthood. ${ }^{10-13)}$ It has been reported that the functional single 
nucleotide polymorphism (SNP; rs6265) of the BDNF gene that produces a valine (Val) to methionine (Met) substitution at codon 66 (Val66Met) affects intracellular trafficking and the activity-dependent secretion of BDNF. ${ }^{14)}$ So far, associations between the Val66Met polymorphism and a variety of psychiatric disorders, such as schizophrenia, ${ }^{15,16)}$ bipolar disorder, ${ }^{17-19)}$ geriatric depression, ${ }^{20)}$ and obsessive compulsive disorder ${ }^{21)}$ have been studied.

In addition, recent studies have presented evidence that BDNF is involved in the pathophysiology of anxiety disorders. The BDNF Val66Met polymorphism is associated with anxiety-related behavior in mice ${ }^{22)}$ and with anxiety-related personality traits in humans. ${ }^{23)}$ Berton et $a l^{24)}$ reported in a study examining the results of a mesolimbic pathway-specific knockdown of the BDNF gene that BDNF was required for the development of social avoidance in response to social defeat stress in mice. Since the avoidance of anxiety-provoking social situations is the core pathology of GSAD, we assumed that the experience-dependent social aversion of mice might be similar to that experienced by GSAD patients.

Based on these findings, we hypothesized that the BDNF gene might be a candidate gene for susceptibility to GSAD. In this study, we investigated the possible association of the val66met polymorphism (rs6265) with GSAD by using a case-control design.

\section{METHODS}

\section{Subjects}

This study was approved by the ethics committee of Kangbuk Samsung Hospital. Seventy-three unrelated patients with GSAD (53 males and 20 females) were recruited from the Department of Psychiatry at Kangbuk Samsung Hospital. All of the subjects were Korean adults who were diagnosed as SAD by well-experienced psychiatrists and met the criteria for GSAD of DSM-IV. We used the Mini-International Neuropsychiatric Interview (MINI), assessed by a research nurse to confirm the diagnosis of GSAD. We excluded the participants who had any other comorbid axis I diagnosis such as major depressive disorder, bipolar disorder, other anxiety disorders, and any kind of psychotic disorders such as schizophrenia and schizoaffective disorder. Anxiety disorder due to general medical condition and substance-induced anxiety disorder were also excluded.

The symptomatic characteristics of these patients were assessed by the Hamilton Anxiety Rating Scale, the Beck Anxiety Inventory, the Retrospective Self Report of
Inhibition, the Spielberger State-Trait Anxiety Inventory (STAI), and the Liebowitz Social Anxiety Scale (LSAS).

The normal control group consisted of 152 healthy volunteers with no past psychiatric history who visited the hospital for their regular health screening. They were also assessed by MINI to ascertain that there was no psychiatric diagnosis.

\section{Genotyping}

Venous blood samples were obtained after the completion of informed consent forms. Genomic DNA was extracted from the white blood cells by standard procedures. The presence of the BDNF G196A (val66met) SNP was assayed by polymerase chain reactions (PCR) using constructed primers (forward: 5'-GGTGAGAAGAGTGATGACCA-3' and reverse: 5'-GCCAGCCAATTCTCTTTTTG-3'). The PCR products were digested at $37^{\circ} \mathrm{C}$ with the restriction enzyme PmaCI (Takara Shuzo Ltd., Kyoto, Japan), which was followed by $2 \%$ agarose gel-electrophoresis, as described previously. ${ }^{25)}$

\section{Data Analysis}

Differences in the frequency distributions of the genotypes, alleles, and allele carriers were examined by cross-tabulation and Pearson chi-square tests using SPSS version 13.0 for Microsoft Windows (SPSS Inc., Chicago, IL, USA). The Hardy-Weinberg equilibrium for the genotype frequencies in the GSAD patients and normal control samples were tested with chi-square tests. The possible effects of the variations in the BDNF polymorphisms on the clinical characteristics of the GSAD patients were examined with ANOVA or $t$-tests by comparing the mean scores determined by the clinical scales. The significance level for the results was set at $p<0.05$.

\section{RESULTS}

All subjects were GSAD patients, whose age ranged from 18 to 60, with a mean (standard diviation, SD) of 31.9 (9.9) years. The mean age (SD) of social anxiety symptom onset was $19.9(9.8)$ and the mean (SD) score of LSAS was 84.4 (26.6).

When comparing the control group and the patients with GSAD group, the mean age (control: $31.61 \pm 9.04$ vs. patients: $31.89 \pm 9.81$ years, $p=0.830]$ was similar, but the sex distribution [male/female: 52/100 (control) and 53/20 (patients), $p<0.001]$ was significantly different. The distributions of the genotypes, alleles, and allele carrier frequencies for the BDNF Val66Met polymorphism in the 
Table 1. Comparison of genotypes, alleles, and Met allele carrier frequencies of the BDNF polymorphism in patients with GSAD and controls

\begin{tabular}{|c|c|c|c|c|c|c|c|c|c|c|}
\hline & \multicolumn{3}{|c|}{ Genotype, n (\%) } & & \multicolumn{2}{|c|}{ Allele, n (\%) } & & \multicolumn{2}{|c|}{ Allele carrier, n (\%) } & \\
\hline & Met/Met & Met/Val & Val/Val & & Met & Val & & Met $(+)$ & Met $(-)$ & \\
\hline Controls $(n=152)$ & $29(19.1)$ & $80(52.6)$ & $43(28.3)$ & $\begin{aligned} \chi^{2} & =0.961 \\
p & =0.619\end{aligned}$ & $138(45.4)$ & $166(54.6)$ & $\begin{array}{r}x^{2}=0.415 \\
p=0.519\end{array}$ & $109(71.7)$ & $43(28.3)$ & $\begin{aligned} \chi^{2} & =0.019 \\
p & =0.889\end{aligned}$ \\
\hline GSAD $(n=73)$ & $18(24.7)$ & $35(47.9)$ & $20(27.4)$ & & $71(48.6)$ & $75(51.4)$ & & $53(72.6)$ & $20(27.4)$ & \\
\hline
\end{tabular}

Met, methionine; BDNF, brain-derived neurotrophic factor; GSAD, generalized social anxiety disorder; Val, valine.

Table 2. Demographic and clinical variables of the three genotype groups of generalized social anxiety disorder patients

\begin{tabular}{|c|c|c|c|c|c|c|c|c|}
\hline & \multicolumn{3}{|c|}{ 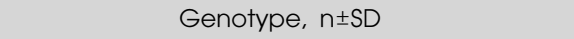 } & \multirow[b]{2}{*}{$F^{*}$} & \multirow[b]{2}{*}{$p^{*}$} & \multicolumn{2}{|c|}{ Allele carrier, $\mathrm{n} \pm \mathrm{SD}$} & \multirow[b]{2}{*}{$p^{\dagger}$} \\
\hline & $\begin{array}{l}\text { Met/Met } \\
(n=18)\end{array}$ & $\begin{array}{l}\text { Met/Val } \\
(n=35)\end{array}$ & $\begin{array}{l}\text { Val/Val } \\
(n=20)\end{array}$ & & & $\begin{array}{l}\text { Met }(+) \\
(n=53)\end{array}$ & $\begin{array}{l}\text { Met }(-) \\
(n=20)\end{array}$ & \\
\hline Age (years) & $32.56 \pm 8.26$ & $31.86 \pm 10.34$ & $31.35 \pm 10.77$ & 0.069 & 0.933 & $32.09 \pm 9.61$ & $31.35 \pm 10.77$ & 0.776 \\
\hline $\begin{array}{l}\text { Age of onset } \\
\text { LSAS score }\end{array}$ & $18.28 \pm 7.14$ & $20.66 \pm 9.93$ & $20.10 \pm 11.64$ & 0.351 & 0.705 & $19.85 \pm 9.08$ & $20.10 \pm 11.64$ & 0.923 \\
\hline Total & $91.88 \pm 28.41$ & $82.52 \pm 25.24$ & $80.00 \pm 27.23$ & 0.961 & 0.388 & $85.98 \pm 26.54$ & $80.00 \pm 27.23$ & 0.444 \\
\hline Fear & $50.56 \pm 14.02$ & $45.69 \pm 12.14$ & $44.06 \pm 14.18$ & 1.203 & 0.307 & $47.44 \pm 12.92$ & $44.06 \pm 14.18$ & 0.366 \\
\hline Avoidance & $39.53 \pm 15.03$ & $34.33 \pm 14.16$ & $35.38 \pm 13.24$ & 0.750 & 0.477 & $36.21 \pm 14.54$ & $35.38 \pm 13.24$ & 0.839 \\
\hline RSRI total score & $73.20 \pm 17.29$ & $73.09 \pm 21.55$ & $79.06 \pm 12.73$ & 0.601 & 0.552 & $73.13 \pm 20.09$ & $79.06 \pm 12.73$ & 0.274 \\
\hline Trait score of STAI & $55.24 \pm 10.43$ & $57.12 \pm 10.52$ & $56.44 \pm 13.08$ & 0.160 & 0.853 & $56.49 \pm 10.42$ & $56.44 \pm 13.08$ & 0.989 \\
\hline HAM-A score & $17.82 \pm 10.43$ & $19.31 \pm 8.57$ & $14.78 \pm 7.77$ & 1.558 & 0.218 & $18.83 \pm 9.14$ & $14.78 \pm 7.77$ & 0.098 \\
\hline
\end{tabular}

Met, methionine; Val, valine; SD, standard deviation; LSAS, Liebowitz Social Anxiety Scale; RSRI, Retrospective Self Report of Inhibition; STAl, Spielberger State-Trait Anxiety Inventory; HAM-A, Hamilton Anxiety Rating Scale.

*One-way ANOVA, IIndependent t-test.

patients and controls are shown in Table 1. Since it has been reported that the Met66 allele is associated with lower activity-dependent secretion of BDNF and abnormal hippocampal function, ${ }^{14)}$ we divided the BDNF Val66Met polymorphism into two groups, namely, carriers of the Met66 allele (Met/Met and Met/Val genotypes) and carriers of the Val/Val genotype. The observed genotype distributions of the patient and control groups did not differ significantly from the expected rate in the Hardy-Weinberg equilibrium (patients: $\chi^{2}=0.58$, degree of freedom [df] $=1, p=0.48$; Controls: $\chi^{2}=0.12, \mathrm{df}=1, p=0.73$ ). There were no significant differences in the frequencies of the genotypes $\left(\chi^{2}=0.96, \mathrm{df}=2, p=0.62\right)$, alleles $\left(\chi^{2}=0.42\right.$, $\mathrm{df}=1, p=0.52)$, or allele carriers $\left(\chi^{2}=0.02, \mathrm{df}=1, p=0.89\right.$ ) between the patients and controls. When the above comparisons were conducted separately by gender, we did not find any differences between the two groups (statistics not shown).

In addition, an analysis was performed to determine the clinical differences arising from the Val66Met polymorphism among the patient groups. An one-way ANOVA between the genotype groups and independent $t$-tests between the Met66 allele carriers and the Val/Val carriers failed to show any significant differences with respect to the clinical variables or to the measured scores on the clin- ical rating scales (Table 2). Furthermore, there were no significant differences in fear and avoidance subscale scores of LSAS as well as the total scores among genotypes.

\section{DISCUSSION}

In this study, the authors investigated association between BDNF Val66Met and GSAD in Korean population. The BDNF has been shown to play an essential role in the avoidance of social defeat stress in experimental animal studies. $^{24)}$ Among the several functional BDNF gene polymorphisms in human, two novel SNPs, C270T and G196A(Val66Met) have been widely investigated. While $\mathrm{C} 270 \mathrm{~T}$ is located in a non-coding region with a promotor function, Val66Met polymorphism is known to affect intracellular trafficking and the activity-dependent secretion of $\mathrm{BDNF}^{14)}$ and most of the papers have concerned the latter. Recently, several studies have reported a possible association between the Met66 allele and anxiety-related personality traits such as harm avoidance. ${ }^{26-28)}$ Based on the previous studies, as the genetic susceptibility and trait interact and mutually affect on the pathophysiology of social anxiety symptom, we hypothesized that BDNF polymorphism would be also related to GSAD. This study 
extends these findings to the clinical situation in order to investigate the possible association between BDNF gene polymorphisms and GSAD. Many genetic association studies only compared the frequencies of the genotypes and alleles in a case-control design. In this study, we also investigated the possible association of the BDNF polymorphism and the severity of anxiety in the GSAD patients by measuring the clinical rating scales.

The two groups, GSAD patients and controls, were not significantly different in genotype distribution, allele frequency, or in the scores of several clinician rating scales of GSAD. Moreover, total and subscale scores of LSAS and the trait scores of the STAI, were compared among genotypes (Table 2), but were found to not differ significantly as well.

However, a possible association between GSAD and the BDNF Val66Met polymorphism cannot be ruled out completely because the gene may play a role as a modulator through interactions with other genes. The BDNF Val66Met polymorphism is known to interact with the serotonin-transporter-linked promoter region (5-HTTLPR) and the dopamine transporter (DAT) VNTR genotype, which modulate the major neurotransmitters, serotonin and dopamine, respectively, and which are closely related to anxiety disorders and influence anxiety-related traits. ${ }^{29,30)}$ In a study of geriatric depression in a Korean population, an altered susceptibility to depression was found not in BDNF gene polymorphisms alone, but in interactions of both the BDNF and 5-HTTLPR genotypes with stressful life events. ${ }^{31)}$ As the genetic association study of GSAD was not much done unlike depressive disorders, the gene and gene interaction should be considered to reveal a significance of some genetic factors on GSAD.

Although genetic factor is regarded as important for the development of SAD, there has been few genetic association studies of SAD. Low extraversion, a predisposing factor of SAD was reported to be associated with catechol-O-methyltransferase gene ${ }^{32)}$ and serotonin transporter promoter (5HTTLPR) gene polymorphism. ${ }^{33)}$ As this studies only studied social anxiety related trait, genome-wide association approaches to studying social anxiety and genetically related phenotypes would offer the opportunity to move beyond candidate genes. Furthermore, although this study did not find any associations between GSAD and the BDNF Val66Met polymorphism, other BDNF polymorphisms may influence the susceptibility or clinical characteristics of GSAD, and so, further studies are needed.

The limitations of this study are as follows. First, the relatively small sample size limits the generalization of our results. These data need to be confirmed in a much larger sample. Second, the sex distributions were significantly different between the patient and control groups. In addition, the male-to-female ratio of $50: 23$ in this study is incongruent with a recent epidemiological study of a community population, ${ }^{34)}$ but in agreement with the result from clinical sample in Korea. ${ }^{35)}$ This result may reflect the genetic characteristics of clinical samples, and community sample study is also needed in the future. Considering that negative results were also obtained when we performed all of the statistical analyses in this study separately by gender, it is not likely that the difference in the sex distribution in our sample would have caused any false-negative results. As we did not investigate genotype of various genes, gene interactions with BDNF gene could not be investigated.

In conclusion, the results of the present study do not support the hypothesis that the BDNF gene might be a candidate gene for susceptibility or severity of GSAD. However, due to many limitations, our negative result should be interpreted carefully and further studies concerning various BDNF polymorphisms with a larger sample may be needed.

\section{REFERENCES}

1. Kessler RC, Berglund P, Demler O, Jin R, Merikangas KR, Walters EE. Lifetime prevalence and age-of-onset distributions of DSM-IV disorders in the National Comorbidity Survey Replication. Arch Gen Psychiatry 2005;62:593-602.

2. Stein MB. An epidemiologic perspective on social anxiety disorder. J Clin Psychiatry 2006;67(Suppl 12):3-8.

3. Schneier FR, Heckelman LR, Garfinkel R, Campeas R, Fallon BA, Gitow A, et al. Functional impairment in social phobia. J Clin Psychiatry 1994;55:322-331.

4. Patel A, Knapp M, Henderson J, Baldwin D. The economic consequences of social phobia. J Affect Disord 2002;68: 221-233.

5. Wittchen HU, Fuetsch M, Sonntag H, Müller N, Liebowitz M. Disability and quality of life in pure and comorbid social phobia. Findings from a controlled study. Eur Psychiatry 2000; 15:46-58.

6. American Psychiatric Association. Diagnostic and Statistical Manual of Mental Disorders. 4th ed. Washington, DC: American Psychiatric Association; 1994.

7. Holt CS, Heimberg RG, Hope DA. Avoidant personality disorder and the generalized subtype of social phobia. $J$ Abnorm Psychol 1992;101:318-325.

8. Mannuzza S, Schneier FR, Chapman TF, Liebowitz MR, Klein DF, Fyer AJ. Generalized social phobia. Reliability and validity. Arch Gen Psychiatry 1995;52:230-237.

9. Stein MB, Chartier MJ, Hazen AL, Kozak MV, Tancer ME, Lander S, et al. A direct-interview family study of generalized social phobia. Am J Psychiatry 1998;155:90-97.

10. Maisonpierre PC, Le Beau MM, Espinosa R 3rd, Ip NY, Belluscio L, de la Monte SM, et al. Human and rat brain- 
derived neurotrophic factor and neurotrophin-3: gene structures, distributions, and chromosomal localizations. Genomics 1991;10:558-568.

11. Maisonpierre PC, Belluscio L, Friedman B, Alderson RF, Wiegand SJ, Furth ME, et al. NT-3, BDNF, and NGF in the developing rat nervous system: parallel as well as reciprocal patterns of expression. Neuron 1990;5:501-509.

12. Lewin GR, Barde YA. Physiology of the neurotrophins. Annu Rev Neurosci 1996;19:289-317.

13. Murer MG, Yan Q, Raisman-Vozari R. Brain-derived neurotrophic factor in the control human brain, and in Alzheimer's disease and Parkinson's disease. Prog Neurobiol 2001;63:71-124.

14. Egan MF, Kojima M, Callicott JH, Goldberg TE, Kolachana $\mathrm{BS}$, Bertolino A, et al. The BDNF val66met polymorphism affects activity-dependent secretion of BDNF and human memory and hippocampal function. Cell 2003;112:257-269.

15. Virgos C, Martorell L, Valero J, Figuera L, Civeira F, Joven $\mathrm{J}$, et al. Association study of schizophrenia with polymorphisms at six candidate genes. Schizophr Res 2001;49:65-71.

16. Muglia P, Vicente AM, Verga M, King N, Macciardi F, Kennedy JL. Association between the BDNF gene and schizophrenia. Mol Psychiatry 2003;8:146-147.

17. Sklar P, Gabriel SB, McInnis MG, Bennett P, Lim YM, Tsan G, et al. Family-based association study of 76 candidate genes in bipolar disorder: BDNF is a potential risk locus. Brain-derived neutrophic factor. Mol Psychiatry 2002; 7:579-593.

18. Neves-Pereira M, Mundo E, Muglia P, King N, Macciardi F, Kennedy JL. The brain-derived neurotrophic factor gene confers susceptibility to bipolar disorder: evidence from a family-based association study. Am J Hum Genet 2002;71: 651-655.

19. Hong CJ, Huo SJ, Yen FC, Tung CL, Pan GM, Tsai SJ. Association study of a brain-derived neurotrophic-factor genetic polymorphism and mood disorders, age of onset and suicidal behavior. Neuropsychobiology 2003;48:186-189.

20. Hwang JP, Tsai SJ, Hong CJ, Yang CH, Lirng JF, Yang YM. The Val66Met polymorphism of the brain-derived neurotrophic-factor gene is associated with geriatric depression. Neurobiol Aging 2006;27:1834-1837.

21. Hall D, Dhilla A, Charalambous A, Gogos JA, Karayiorgou M. Sequence variants of the brain-derived neurotrophic factor (BDNF) gene are strongly associated with obsessivecompulsive disorder. Am J Hum Genet 2003;73:370-376.

22. Chen ZY, Jing D, Bath KG, Ieraci A, Khan T, Siao CJ, et al. Genetic variant BDNF (Val66Met) polymorphism alters anxiety-related behavior. Science 2006;314:140-143.

23. Lang UE, Hellweg R, Kalus P, Bajbouj M, Lenzen KP, Sander $\mathrm{T}$, et al. Association of a functional BDNF polymor- phism and anxiety-related personality traits. Psychopharmacology (Berl) 2005;180:95-99.

24. Berton O, McClung CA, Dileone RJ, Krishnan V, Renthal $\mathrm{W}$, Russo SJ, et al. Essential role of BDNF in the mesolimbic dopamine pathway in social defeat stress. Science 2006; 311:864-868.

25. Itoh K, Hashimoto K, Kumakiri C, Shimizu E, Iyo M. Association between brain-derived neurotrophic factor 196 G/A polymorphism and personality traits in healthy subjects. Am J Med Genet B Neuropsychiatr Genet 2004;124B:61-63.

26. Kim SJ, Cho SJ, Jang HM, Shin J, Park PW, Lee YJ, et al. Interaction between brain-derived neurotrophic factor Val66Met polymorphism and recent negative stressor in harm avoidance. Neuropsychobiology 2010;61:19-26.

27. Jiang X, Xu K, Hoberman J, Tian F, Marko AJ, Waheed $\mathrm{JF}$, et al. BDNF variation and mood disorders: a novel functional promoter polymorphism and Val66Met are associated with anxiety but have opposing effects. Neuropsychopharmacology 2005;30:1353-1361.

28. Montag C, Basten U, Stelzel C, Fiebach CJ, Reuter M. The BDNF Val66Met polymorphism and anxiety: support for animal knock-in studies from a genetic association study in humans. Psychiatry Res 2010;179:86-90.

29. Terracciano A, Tanaka T, Sutin AR, Deiana B, Balaci L, Sanna S, et al. BDNF Val66Met is associated with introversion and interacts with 5-HTTLPR to influence neuroticism. Neuropsychopharmacology 2010;35:1083-1089.

30. Hünnerkopf R, Strobel A, Gutknecht L, Brocke B, Lesch $\mathrm{KP}$. Interaction between BDNF Val66Met and dopamine transporter gene variation influences anxiety-related traits. Neuropsychopharmacology 2007;32:2552-2560.

31. Kim JM, Stewart R, Kim SW, Yang SJ, Shin IS, Kim YH, et al. Interactions between life stressors and susceptibility genes (5-HTTLPR and BDNF) on depression in Korean elders. Biol Psychiatry 2007;62:423-428.

32. Stein MB, Fallin MD, Schork NJ, Gelernter J. COMT polymorphisms and anxiety-related personality traits. Neuropsychopharmacology 2005;30:2092-2102.

33. Arbelle S, Benjamin J, Golin M, Kremer I, Belmaker RH, Ebstein RP. Relation of shyness in grade school children to the genotype for the long form of the serotonin transporter promoter region polymorphism. Am J Psychiatry 2003;160: 671-676.

34. Cho MJ, Kim JK, Jeon HJ, Suh T, Chung IW, Hong JP, et al. Lifetime and 12-month prevalence of DSM-IV psychiatric disorders among Korean adults. J Nerv Ment Dis 2007; 195:203-210.

35. Lee SH, Shin YC, Oh KS. A clinical study of social phobia for 10 years. J Korean Neuropsychiatr Assoc 1994;33:305312 . 